\title{
Distance estimation in vista space
}

\author{
S. Oliver Daum and Heiko Hecht \\ Johannes Gutenberg-Universität Mainz, Mainz, Germany
}

\begin{abstract}
Although distance estimation has been extensively studied in the laboratory, our ability to judge large distances in the field is not well researched. We challenge the notion that large distances are uniformly underestimated. We presented different targets to observers at distances ranging from 25 to $500 \mathrm{~m}$ to obtain egocentric distance judgments in natural environments. Three experiments showed that observers tend to underestimate distances below $75 \mathrm{~m}$ in a large open field, whereas they overestimate farther distances. Both the eye height of the observer and the size of the target also influenced distance estimation. We conclude that the notion of a uniform vista space has to be reconceived.
\end{abstract}

Within action space, egocentric distances can be judged with approximate accuracy (e.g., Kuroda, 1971; Teghtsoonian \& Teghtsoonian, 1970), whereas exocentric distance judgments often reveal dramatic underestimation, which tends to be more or less extreme depending on the action context (e.g., Loomis, Da Silva, Philbeck, \& Fukusima, 1996; Wagner, 1985). In contrast, surprisingly little is known about the human ability to estimate distances beyond one's immediate action space. It is sometimes thought that perceptual space is compressed, causing large distances to be generally underestimated (e.g., Hecht, van Doorn, \& Koenderink, 1999), but this impression does not seem to be shared by expert opinion. One might think not only that land surveyors, hunters, forest officials, hikers, and golfers have developed expert knowledge in this domain, but also that psychological evidence has been collected to back up the experts. The latter evidence is, in fact, rather meager. The present study was designed to fill this gap. We first will discuss existing space taxonomies, arguing that they differentiate well within action space (up to egocentric distances of 8 or, maximally, $30 \mathrm{~m}$ ) but that they fail to properly differentiate vista space (beyond $30 \mathrm{~m}$ ). We are interested in the vista space realm and maintain that distance estimation in vista space is not uniform. We then will mention the existing anecdotal and the scant scientific evidence, after which we will report two distance estimation experiments conducted in the open field and one conducted in the laboratory. The experiments suggested that there is a qualitative step within vista space, so that overestimation is facilitated beyond approximately $75-100 \mathrm{~m}$.

\section{Action Space and Vista Space}

Grüsser $(1978,1983)$ divided perceptual space into the two major regions of personal and extrapersonal space. Extrapersonal space was further subdivided into grasping space (and instrumental grasping space), near-distant action space (up to distances of approximately $8 \mathrm{~m}$, depending on training, etc.), far-distant action space, and the visual background (such as the sky). In a similar fashion, Cutting and Vishton (1995) subdivided space into three circular, egocentric regions, which they called personal space (limited to $2 \mathrm{~m}$ ), action space (up to a radius of $30 \mathrm{~m}$ ), and vista space (beyond about $30 \mathrm{~m}$ ). These space taxonomies are particularly useful because they suggest that perceptual-motor precision decreases with the radius. Accordingly, it can be assumed that distance perception modes differ as a function of distance from the observer.

Personal space and near-distant action space in the sense of Grüsser $(1978,1983)$, or personal space in Cutting and Vishton's (1995) terms, have been explored at length over the last 150 years (e.g., Gilinsky, 1951; Gogel, 1964; Hauck, 1875; Loomis, Da Silva, Fujita, \& Fukusima, 1992; Lukas, 1996). Barring consistent and well-researched minor distortions, distance estimation is remarkably accurate in personal space, where accommodation, convergence, and retinal disparity are informative. The same holds for Grüsser's so-called near-distant action space. Far-distant action space beyond $8 \mathrm{~m}$ away from the observer, in contrast, can be characterized by spatial compression of the sagittal dimension. This compression is modest when egocentric distance is to be judged, whereas it can lead to overestimations of up to $100 \%$ when exocentric judgments have to be made (Amorim, Loomis, \& Fukusima, 1998; Hecht et al., 1999; Loomis et al., 1996; Norman, Todd, Perotti, \& Tittle, 1996; Toye, 1986; Wagner, 1985). That is, distances along the observer's line of sight can be grossly underestimated, whereas lateral distances do not suffer the same fate.

The underestimation of egocentric distance estimation in action space depends considerably on the method. Verbal estimate methods tend to be less accurate than actionbased estimation methods, such as blindfolded walking. For instance, Andre and Rogers (2006) reported that verbal

H. Hecht, hecht@uni-mainz.de 
estimations of egocentric distance were usually too short at distances greater than several meters (up to $90 \mathrm{ft}$ ), whereas blindfolded walking to previously viewed targets was consistently more accurate than verbal reports. Other methods, such as walking to a target by triangulation, typically produce dramatic underestimation of distance, often by as much as $50 \%$-in particular, when virtual environments are used (see Thompson, Willemsen, Gooch, \& CreemRegehr, 2004). Note, however, that estimates within the visual modality can produce both over- and underestimation as a function of the psychophysical method that is applied (see the nice juxtaposition of methods by Da Silva, 1985).

The notion of sagittal compression in action space is consistent with findings that slope - or to be precise, geographical slant - is overestimated in this distance range of up to 30 m (e.g., Proffitt, Bhalla, Gossweiler, \& Midgett, 1995). Likewise, in a more anecdotal fashion, Baden-Powell (1944) suggested that merely looking uphill or downhill leads to underestimation of distance. Sagittal compression in action space is also consistent with the finding that right angles of buildings are perceptually flattened in action space (Hecht et al., 1999). If these findings are taken together, the case for compression is fairly strong for action space, whereas it is anything but clear for vista space.

Distance estimation capabilities in vista space are hard to assess because the visual system has to rely on pictorial cues, whose availability may change dramatically as a function of terrain, lighting, weather, and so forth. However, once such particular effects are removed, is there evidence for spatial compression, as has been found in action space? To answer the question, we first will summarize the known pictorial distance cues and then will consider some nonpictorial cues, such as fatigue.

\section{Pictorial Distance Cues in Vista Space}

We know a little about the role of situational visual cues from sources other than anecdotal lore, and, where we do, the anecdotal evidence, gleaned from such sources as field manuals and military handbooks (e.g., Allekseenko, 1989; Ivanov, 1969; Stockfisch, 2006), has often been consistent with the results of empirical studies (e.g., Ross, 1974; Ross \& Plug, 2002). Both sources have suggested an underestimation of distance on uniform and empty terrain, which has ordinarily been used in outdoor experiments.

Some of the earliest reported experiments of distance estimation in a large-scale outdoor environment were conducted by E. J. Gibson and Bergman (1954) and E. J. Gibson, Bergman, and Purdy (1955) on a large grassy athletic field $(446 \times 121 \mathrm{~m})$. Distances ranging from 48 to $361 \mathrm{~m}$ had to be judged. The errors showed predominantly an underestimation of distance, with a strange tendency to overestimate farther targets. In a similar experiment, in which the method of fractionation was used, Purdy and Gibson (1955) found fairly accurate performance for distances between 23 and $229 \mathrm{~m}$. Galanter and Galanter (1973) reported that far distances of boats were overestimated at distances beyond 100 yards. There also was a tendency toward increasing errors when large distance stretches were fractionated. More recently, Plumert, Kearney, Cremer, and Recker (2005) found that distances between 20 and
$120 \mathrm{ft}$ were underestimated when, using an indirect assessment method, they measured the time observers thought it would take to walk to the target.

In a study for the Army Personnel Research Establishment, Bee (1991) found some different results. The target was a three-quarter-ton Land-Rover SUV whose distance had to be estimated at 10 different locations ranging from 500 to $2,130 \mathrm{~m}$ away from the observer in an open area. First, it was found that the relative error increased significantly with the actual distance. More interestingly, the general tendency to underestimate was found only for the near distance, whereas at distances over $1,500 \mathrm{~m}$, a general tendency toward overestimation was observed. In a similar experiment, Fine and Kobrick (1983) used a familiar military truck as a target that had to be judged at observer distances ranging from 600 to $1,550 \mathrm{~m}$. The truck was placed on a large, flat, and grassy area adjacent to an airfield runway against a distant background of low hills and trees. They found that the average judgments were accurate up to $1,100 \mathrm{~m}$, but beyond that, both experienced and untrained observers tended to overestimate distance, while their performance became rather variable. Interestingly, only the experienced group of subjects consistently overestimated the actual distance.

From these findings, we surmise that distance estimation in vista space cannot be described as uniform spatial compression, as is the case for action space. Instead, near vista space appears to be like action space in that respect, whereas far vista space above a few hundred meters appears to be dilated. Before we test this hypothesis, we will briefly consider those potentially confounding pictorial cues that might distort distance estimates.

Aerial perspective. The visibility of a far object is often reduced by the atmosphere (Ross, 1974, pp. 49ff). Atmospheric particles cause blur that increases with distance. In clear atmosphere, objects also acquire a blue tint in the distance. Very far objects, like mountains, may appear to be quite near (J. J. Gibson \& Flock, 1962) when the air is unusually clear. Astronauts have complained that it is difficult to estimate the distance of mountains on the moon, presumably because the absence of an atmosphere perspective. There is evidence that the reduction in brightness and color contrast of distant objects acts as a distance cue. Ross $(1967,1975)$ showed, with a group of students, that they overestimated distances in fog, in comparison with a group of students who had to judge the same distances in clear weather. The overestimation tended to increase with distance. Size judgments were similarly affected by fog and under water; both made objects appear larger. It was concluded that the overestimation of size in fog and under water is due to a relative overestimation of distance.

Texture gradient. According to J. J. Gibson (1950), the density of texture elements, relative to the maximal and minimal density over the entire image, furnishes information about distance at any point on a receding surface. A well-structured terrain should improve distance estimates, and the observer's eye height should matter, producing overestimation of distance in prone positions, as compared with the upright. This effect has found its way into field manuals (see Baden-Powell, 1944; Stockfisch, 2006). 
Also, Higashiyama (1996) claimed that distance estimation changes as a function of how strongly compression of the ground texture occurs. The lower the observer's eye height, the stronger the compression and, consequently, the longer the judged distances (Higashiyama \& Ueyama, 1988). The visual representation of the near ground is thought to serve as an anchor early on in the surface representation process (Wu, Ooi, \& He, 2004) and could thus explain an overestimation of distance by prone observers. This is in keeping with the proposal by J. J. Gibson (1950) that the ground surface is used as a reference frame for coding the location of an object. Also, Sinai, Ooi, and He (1998) found that discontinuities in surface texture (gaps of varying depth and widths) led to distance overestimation in a blindfolded-walking and a perceptual-matching task. In the same vein, Lappin, Shelton, and Rieser (2006) described the influence of context on visually perceived distances. The accuracy and the precision of perceived distance depended on subtle properties of the surrounding environment. Interestingly, perceived gap crossability and depth also depended on the observer's direction of gaze (Jiang \& Mark, 1994). This finding suggests that extraretinal factors, such as gaze direction, have to be taken seriously in the context of distance estimation.

Geographical and optical slant. J. J. Gibson and Cornsweet (1952; J. J. Gibson, 1979) distinguished optical from geographical slant. Optical slant is specified in relation to the line of sight from the point of observation to the surface. Geographical slant is defined as an inclination relative to the surface of the earth - the horizontal ground plane. If optical slant interacts with the perception of geographical slant, the uphill view of a slope should look steeper than a downhill view, due to the foreshortening of the distance to the bottom (Ross, 1974, pp. 70ff). Slope illusions can occur because of the misperception of bodily orientation or misleading contextual cues (see Bressan, Garlaschelli, \& Barracano, 2003). The tendency to overestimate the steepness of a frontal slope is strong even when an observer is in contact with the slope. This, in turn, could influence distance estimation.

Another important fact is the interaction between single terrain qualities when slopes and planar areas join in a natural geographic scene that contains hollows or canyons. Peaks that are level with an observer's position appear considerably higher, and all points on opposite mountains are raised correspondingly (Ross, 1974, p. 68).

\section{Action Cues in Vista Space}

Effort. What complicates the issue is that distance may be coded in effort units rather than optical units (Kammann, 1967; Loomis et al., 1996). Ross (1974, p. 116) observed that a distance seems longer if great effort is involved, if the route is new, or if the traveler is tired or anxious. A distance should, accordingly, appear longer when walked than when run. Okabe, Aoki, and Hamamoto (1986) provided partial evidence for these conjectures by showing that a traversed distance was overestimated not only in uphill walking, but also in downhill walking, for both children and adults. Because the effects of effort and texture oppose one another on slanted terrain, they supposed that overestimation in uphill walking is caused mainly by physical effort, whereas overestimation in downhill walking is caused mainly by the effort of exercising caution. Consistent with the effort hypothesis, Proffitt and colleagues (Bhalla \& Proffitt, 1999; Creem \& Proffitt, 1998) showed that hills appear steeper and distances farther when one is wearing a heavy backpack or is fatigued after an exhausting run, or when one is in ill health. Even more surprisingly, Proffitt, Stefanucci, Banton, and Epstein (2003) found that merely anticipated effort suffices to inflate distance estimates. Witt, Proffitt, and Epstein (2004) showed that, as the effort associated with walking increased, perceived distances increased if the observer intended to walk, but not if the observer's intention was to throw an object, which indicates that the effect of effort is contingent upon anticipated or actual physical activity. These findings, however, have been challenged empirically, as well as on theoretical grounds, by Hutchinson and Loomis (2006a), who were not able to replicate these results and who argued that response modality may explain some of the differences (see also Hutchinson \& Loomis, 2006b; Proffitt, Stefanucci, Banton, \& Epstein, 2006a, 2006b). The results certainly underline the complexity of distance estimation.

Expert opinion. Experts in the domain of vista space distance estimation appear to have rather clear-cut guidelines, which remain at a qualitative level. For instance, army field manuals point out conditions that lead to systematic estimation errors (see Allekseenko, 1989; Ivanov, 1969; Stockfisch, 2006, part C, p. 223). Soldiers tend to underestimate distances when the targets are clearly visible, the air is clear, the sun is at their back, or the terrain is not fully visible or uniform (e.g., in snow or across water surfaces). Interestingly, the temporal proximity of combat is also taken to cause underestimation of distance. Overestimation occurs if the targets are barely visible, the observer is lying on the ground, the air glitters, or the sun is facing the observers. Overestimation is also taken to occur in foggy or bad weather, during dusk or dawn, and in forested areas. This becomes relevant, for instance, when one is aiming at flying targets. Their speed and distance have to be judged with high accuracy in order to choose the appropriate lead angle (Watson, 1982, pp. 75ff).

These insights mostly concur with remarks by Ross (1974, p. 49), who, among others, quotes Baden-Powell's Scouting for Boys (1944) handbook as follows:

Objects appear nearer than they really are: First when the light is bright and shining on the object; secondly when looking across water or snow, or looking uphill or down. Objects appear farther off when in the shade; across a valley; when the background is of the same color; when the observer is lying down or kneeling; when there is a heat haze over the ground. (p. 40)

To summarize, in vista space, pictorial cues gain importance. Among these are object occlusion, texture, amount of detail, color contrast, atmospheric conditions, illumination, aerial perspective, and so forth. These cues are moderated by the observer's position and his or her experience in distance estimation. The few existing empirical studies point to a potential anisotropy between action space and 
vista space. The near region of vista space, up to $100 \mathrm{~m}$ or so, appears to behave like action space, whereas larger distances might even produce distance overestimation. We sought to evaluate whether this is, in fact, the case. We attempted to hold constant as many variables as possible and to investigate whether the position of the observer (prone, upright, and raised), the size of unfamiliar targets (small, medium-sized, and large), and the area conditions (upand downhill slope and planar surface) would influence the estimation of distances ranging from $25 \mathrm{~m}$ up to $460 \mathrm{~m}$ in a large open field. We were also interested in possible differences between natural full-cue viewing conditions and a laboratory setting. Thus, two field experiments and one experiment in the laboratory were conducted.

\section{EXPERIMENT 1 Distance Estimation in the Open Field}

We chose a countryside area consisting of gentle hills strewn with forest and meadows as a test site. The expanse of the field was approximately $750 \mathrm{~m}$, without salient landmarks or orientation points. The site allowed us to position targets at distances ranging from 25 to $460 \mathrm{~m}$ in such a way that houses, cars, or other familiar objects did not come into view. We chose to test in clear daylight. Several flat areas and a gentle hill with a constant slope of approximately $300 \mathrm{~m}$ in length and an angle of $12^{\circ}$ were selected. The flat terrain was a wide, open field covered with grass and bushes and surrounded by trees. On the basis of the existing anecdotal knowledge and extrapolating from action space effects into vista space, four different hypotheses were generated. First, the farther the target is presented from the observer, the greater the distance estimation error should become. Second, the eye height of an observer serves as a utility to interpret perspective foreshortening in the context of height estimates of objects (Wraga \& Proffitt, 2000). The effectivity of this cue might be confined to eye heights between $20 \%$ and $250 \%$ of the observer's normal standing eye height. This cue may well be at work when judging distances. Thus, prone observers should produce farther distance judgments than should upright observers. Third, the distance between observers and targets will be estimated as shorter in a planar area than in an uphill area. Fourth, according to a retinal size heuristic, small targets should be judged to be farther away than large targets.

\section{Method}

Observers. Fifteen observers participated in this experiment. Fourteen of them volunteered for the first part of the experiment, and 11 for the second. Their ages ranged from 23 to 75 years, with an average age of 51 years. Two subjects were female. All had normal or corrected-to-normal vision and were naive with respect to the purpose of the study.

Stimuli and Design. The experiment was conducted outdoors on a large open field in a planar and an uphill area condition. In both conditions, no salient landmarks or orientation points were given. Four distances $(54,217,335$, and $460 \mathrm{~m})$, three target sizes of small $(0.5 \times 0.5 \mathrm{~m})$, medium-sized $(0.75 \times 0.75 \mathrm{~m})$, and large $(1.0 \times$ $1.0 \mathrm{~m}$ ) targets, and the two observer positions (prone and upright) were fully crossed, resulting in 24 estimates. These trials were administered in a separate counterbalanced block in an uphill condi- tion. However, in the uphill block, the observers had to judge only two distances (57 and $225 \mathrm{~m}$ ). Within each block, the targets were positioned in randomized orders. For a given distance, the targets were placed at varying lateral positions to prevent effects of particular landscape cues or habituation. Each distance required 6 estimations ( 3 target sizes $\times 2$ observer positions).

The targets were square wooden boards scaled to different sizes. The boards, $8 \mathrm{~mm}$ thick, were fixed, respectively, on a wooden frame of appropriate size. All targets featured a black painted cross on a white background.

For the distance measurement, a Leica LAF 800 Rangemaster laser distance finder was used, as well as a U.S. Ranger Import compass to keep a record of the position of the sun and a Canon Digital Ixus 500 camera for documentation of the trials.

Procedure. A distance of $10 \mathrm{~m}$ was presented to give the subjects a reference by which to calibrate their judgments. For this purpose, two piles of car tires were used, which stood $2 \mathrm{~m}$ apart and $10 \mathrm{~m}$ away from the observer. The observers had to estimate the egocentric distance to the tires, as well as the exocentric distance between the tires, in a verbal report. The observers were then informed about these calibration distances. Per trial, the experimental targets were presented in pseudorandom order blocked for prone and upright postures. The observers had to estimate the distance along the line of sight. In the experimental trials, no feedback was given. To prevent the observers from influencing one another, they were not allowed to talk during the experiment and had to give their distance estimation by writing it down on a questionnaire with one page for each judgment. They were allowed to take as long as they needed for their judgment. After each distance estimate, a difficulty score of the estimate had to be given on a 10-point rating scale.

After a given target had been judged, it was removed by one assistant while another assistant positioned the next target. During this reconstruction, the observers had to turn around $180^{\circ}$, so they never saw the assistants and were unable to use them as a distance cue. Twenty-four trials of distance estimation were performed in the planar area, 12 trials in the uphill area. Between the planar and uphill locations, a foot march of $5 \mathrm{~min}$ was necessary. To ensure that the observers did not see the targets, they were transported separately. After transport, the experimenter returned to the observers and led them to the new experimental area.

\section{Results and Discussion}

The average distance judgments revealed a general tendency toward overestimation for all distances, all observer positions, and all target sizes (see Table 1). The relative overestimation increased almost linearly with distance, with the exception of a plateau between 217 and $335 \mathrm{~m}$ (Figure 1). The magnitude of overestimation for targets on the two uphill slopes was less pronounced for the short distance ( $20 \%$ overestimation of the $54-\mathrm{m}$ target) than for the $217-\mathrm{m}$ target ( $92 \%$ overestimation).

A repeated measures ANOVA on relative distance error (4 levels of distance $\times 3$ levels of target size $\times 2$ levels of observer position) revealed significant effects of actual distance for the planar area $[F(3,39)=18.503, p<.001, \varepsilon=$ .586]. Distance estimation uphill ( 2 levels of distance $\times 3$ levels of target size $\times 2$ levels of observer position) also reached significance $[F(1,10]=5.593, p=.040, \varepsilon=$ 1.000]. No significant effects of observer position could be found. Contrary to our expectations, prone observer positions did not differ from upright postures. Although there was a tendency in the hypothesized direction, the difference between planar and uphill areas was not significant ( 2 levels of area condition $\times 2$ levels of distance $\times 3$ levels of target size $\times 2$ levels of observer position) $[F(1,9)=0.246$, 
Table 1

\begin{tabular}{|c|c|c|c|c|c|c|c|}
\hline \multirow{3}{*}{$\begin{array}{l}\text { Distance } \\
\text { (m) }\end{array}$} & \multirow[b]{3}{*}{ Position } & \multicolumn{6}{|c|}{ Target Size } \\
\hline & & \multicolumn{2}{|c|}{$0.5 \times 0.5$} & \multicolumn{2}{|c|}{$0.75 \times 0.75$} & \multicolumn{2}{|c|}{$1.0 \times 1.0$} \\
\hline & & $M$ & $S D$ & $M$ & $S D$ & $M$ & $S D$ \\
\hline \multicolumn{8}{|c|}{ Plane } \\
\hline 54 & $\begin{array}{l}\text { Prone } \\
\text { Upright }\end{array}$ & $\begin{array}{l}72.857 \\
75.000\end{array}$ & $\begin{array}{l}24.939 \\
17.541\end{array}$ & $\begin{array}{l}71.500 \\
72.500\end{array}$ & $\begin{array}{l}26.399 \\
30.619\end{array}$ & $\begin{array}{l}67.143 \\
63.929\end{array}$ & $\begin{array}{l}21.725 \\
19.727\end{array}$ \\
\hline 217 & $\begin{array}{l}\text { Prone } \\
\text { Upright }\end{array}$ & $\begin{array}{l}445.714 \\
372.857\end{array}$ & $\begin{array}{l}129.538 \\
120.284\end{array}$ & $\begin{array}{l}355.000 \\
392.857\end{array}$ & $\begin{array}{r}84.830 \\
126.881\end{array}$ & $\begin{array}{l}362.857 \\
396.429\end{array}$ & $\begin{array}{r}97.225 \\
111.742\end{array}$ \\
\hline 335 & $\begin{array}{l}\text { Prone } \\
\text { Upright }\end{array}$ & $\begin{array}{l}610.714 \\
542.857\end{array}$ & $\begin{array}{l}171.754 \\
130.880\end{array}$ & $\begin{array}{l}590.714 \\
557.143\end{array}$ & $\begin{array}{l}163.776 \\
163.915\end{array}$ & $\begin{array}{l}595.000 \\
633.571\end{array}$ & $\begin{array}{l}164.445 \\
128.339\end{array}$ \\
\hline 460 & $\begin{array}{l}\text { Prone } \\
\text { Upright }\end{array}$ & $\begin{array}{l}1,125.000 \\
1,039.286\end{array}$ & $\begin{array}{l}134.092 \\
176.699\end{array}$ & $\begin{array}{r}1,019.286 \\
911.429\end{array}$ & $\begin{array}{l}131.761 \\
156.395\end{array}$ & $\begin{array}{l}828.571 \\
892.857\end{array}$ & $\begin{array}{l}176.193 \\
114.114\end{array}$ \\
\hline \multicolumn{8}{|c|}{ Uphill } \\
\hline 57 & $\begin{array}{l}\text { Prone } \\
\text { Upright }\end{array}$ & $\begin{array}{l}69.091 \\
75.091\end{array}$ & $\begin{array}{l}18.950 \\
20.251\end{array}$ & $\begin{array}{l}68.091 \\
66.364\end{array}$ & $\begin{array}{l}22.483 \\
31.232\end{array}$ & $\begin{array}{l}63.636 \\
67.727\end{array}$ & $\begin{array}{l}25.406 \\
19.412\end{array}$ \\
\hline 225 & $\begin{array}{l}\text { Prone } \\
\text { Upright }\end{array}$ & $\begin{array}{l}435.000 \\
495.455\end{array}$ & $\begin{array}{l}229.031 \\
311.010\end{array}$ & $\begin{array}{l}447.273 \\
366.818\end{array}$ & $\begin{array}{l}207.224 \\
189.160\end{array}$ & $\begin{array}{l}451.818 \\
362.364\end{array}$ & $\begin{array}{l}194.516 \\
197.700\end{array}$ \\
\hline
\end{tabular}

$p=.650, \varepsilon=.100]$. The size of the presented target had an influence on the estimation of distance in the planar area condition, whereas for estimating uphill, the effect failed to

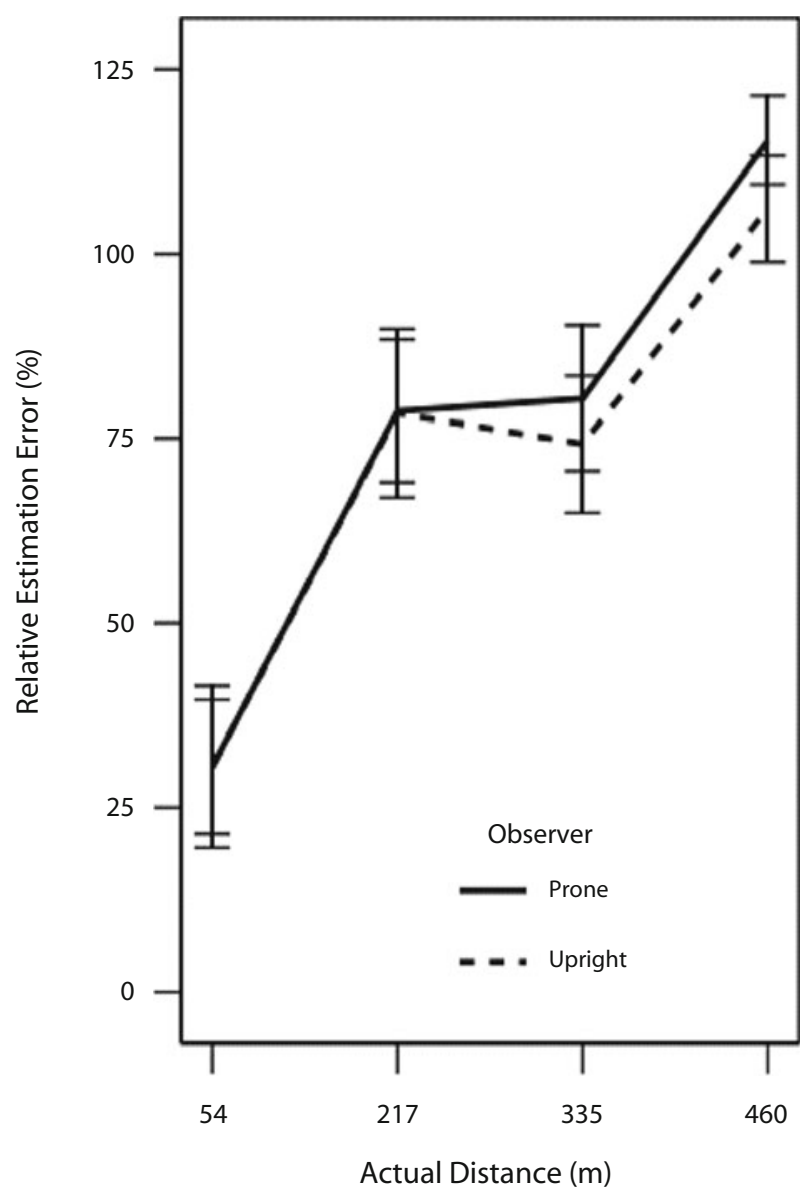

Figure 1. Experiment 1: Average estimation errors expressed in percentages of actual distances for prone and upright observer positions in planar terrain. Error bars represent standard errors of the means. reach significance [planar, $F(2,26)=7.145, p=.009, \varepsilon=$ .732 ; uphill, $F(2,20)=3.094, p=.088, \varepsilon=.733]$. As was expected, the smaller targets were estimated to be farther away than the larger targets. A significant distance $\times$ target size interaction was found for the planar area condition, but not for distance estimation uphill [planar, $F(6,78)=$ 5.123, $p=.003, \varepsilon=.559$; uphill, $F(2,20)=0.376, p=$ $.631, \varepsilon=.740]$. A further significant target size $\times$ observer position interaction was found for the planar area condition, as well as for the uphill condition [planar, $F(2,26)=4.509$, $p=.036, \varepsilon=.706$; uphill, $F(2,20)=3.986, p=.048, \varepsilon=$ .786]. The small targets were estimated to be farther away in prone than in upright postures, whereas this posture effect was absent for medium-sized and large targets. Note that the overall effect size of posture was very small. Across all distances, distance judgments did not vary significantly with posture (Cohen's $d=.20$ ), and for the largest distance of $460 \mathrm{~m}$, judgments in the prone position were larger $[t(13)=3.74, p=.003]$, which also constitutes a small effect (Cohen's $d=.38$ ).

Inconsistent with our hypothesis, no effect of terrain was found. Targets on the flat terrain and on the uphill slope were overestimated to comparable extents. Maybe an inspection of the terrain while the observers were walking up to the observation point was responsible for this outcome. In sum, it was shown that overestimation increases with actual distance. The larger targets were estimated to be nearer. This effect of target size points to a bigger-nearer heuristic-in particular, in the planar area condition for the prone observer. Most surprisingly, we could not detect a trace of spatial compression even for the closest distances. The vista space task appears to have caused a strong overestimation of distance, which needs to be further explored.

\section{EXPERIMENT 2}

\section{Replication in the Laboratory}

As ecologically sound as the outdoor viewing scenario of Experiment 1 may have been, the number of stimuli that could be presented was rather limited. We chose to 
replicate the experiment in the laboratory with a larger range of conditions and in a more controlled setting, using photographs of the same sites and targets as those used in Experiment 1, supplemented by more uphill slopes, downhill slopes, and observer positions. We also changed the viewing position in front of the large projection screen.

We hypothesized that we would find the same pattern of results as in Experiment 1, perhaps with the exception that very far targets might get too close to the resolution limits of the projection system. Also, distance estimates for targets in the planar areas should be shorter than those in uphill areas and possibly larger than those in downhill areas. For economical reasons, we wanted to test observers in groups, but we were concerned that the observers' viewing positions in front of the screen might interact with distance estimation. Thus, we deliberately compared oblique with centered viewing positions.

\section{Method}

Observers. Twenty-eight observers participated in this experiment. Their ages ranged from 18 to 45 years, with an average age of 24 years. Twenty-two of the 28 subjects were female. All the observers were psychology students at the Johannes Gutenberg University of Mainz, Germany. All had normal or corrected-to-normal vision and were naive with respect to the purpose of the study.

Stimuli and Design. The experiment was conducted in the Virtual Reality Laboratory at the Psychological Institute of Johannes Gutenberg University of Mainz. The experimental materials were 270 pictures, taken from the same natural field areas as those used in Experiment 1 . In all the photographs, salient landmarks or orientation points were avoided. Four distances (54, 217, 335, and $460 \mathrm{~m})$ and three target sizes (small, $0.50 \times 0.50 \mathrm{~m}$; medium-sized, $0.75 \times$ $0.75 \mathrm{~m}$; and large, $1.0 \times 1.0 \mathrm{~m}$ ) were used, as well as the three camera positions corresponding to a prone, upright, and raised $(280 \mathrm{~cm}$ above the ground) observer. In the uphill and downhill slope conditions, only three target distances could be used $(57,225$, and $335 \mathrm{~m})$. The downhill condition involved the same hill as that used for the uphill estimations, which had already been used in Experiment 1. For the photographs, a Canon Ixus 50 digital camera with 5 megapixels and a focal length of $5.8 \mathrm{~mm}$ was used. Note that the small CCD chip of the camera renders this focal length roughly equivalent to the slight wide angle $(35 \mathrm{~mm})$ of a standard $35-\mathrm{mm}$ camera. The pictures were taken from the left and right borders of the areas, as well as from the center, in order to provide variable views on targets at a given distance.

Procedure. The observers were distributed into 12 groups made up maximally of 4 subjects. To calibrate their judgments, a reference picture was presented that contained two rain barrels, $2 \mathrm{~m}$ apart and $10 \mathrm{~m}$ away from the camera. The observers had to estimate the egocentric distance to the barrels, as well as the exocentric distance between the tires, in a verbal report. After the estimate, the observers received feedback about the actual distance of the barrels. The photographic stimuli were presented in separate random orders on a large rear projection screen. The randomization software (irfanView 3.97) ran on a Dell Precision 360, with a Pentium P IV Processor (2.9 GHz), 1 GB of RAM, and a 256-MB nVidia QuadroFX 3000 graphic card. The screen was $260 \mathrm{~cm}$ wide and $195 \mathrm{~cm}$ tall. Two JVC D-ILA DLA SX21 projectors with a resolution of $1,400 \times 1,050$ pixels were used. The observers were seated at a distance of $249 \mathrm{~cm}$ from the center of the screen, so that the visual angle of a target at a given distance matched exactly its outdoor equivalent in Experiment 1 . We verified this distance with visual angle measurements. The theoretical equivalence based on the specifications of the camera was calculated to require an observer distance from the screen of $262 \mathrm{~cm}$. The width of the entire scene amounted to a visual angle of $96.2^{\circ}$. For each group, 1 observer sat centered, and 2 were placed $13^{\circ}$ to the left and right, respectively, as measured from the center of the screen. As in Experiment 1, the observers had to give their distance judgments by questionnaire, without the possibility that the other subjects were able to take a look at the answer. The experimenter advanced to the next photograph when all the observers had made their judgments - typically, after $20 \mathrm{sec}$.

\section{Results and Discussion}

Although the results were similar to those of the field experiment, there were some notable differences (see Figure 2). A similar tendency toward overestimation in vista space was found; however, the effect appeared only at distances above $200 \mathrm{~m}$. As before, the relative overestimation was found to increase with distance in flat terrain, but much less so on uphill or downhill slopes. The slopes showed only nonsignificant trends in this direction. In the laboratory experiment, standard deviations and individual differences among observers were much larger.

Against the general trend, some observers tended to overestimate near and to underestimate far distances. In the natural environment in which the photographs were taken, the observers behaved rather homogeneously. This suggests that pictures of vista space are not treated similarly by all observers, which makes the use of pictures rather problematic. A closer look at the individual differences revealed that nearly all the observers, when asked after the experiment, had rather false ideas about the actual size of the targets. This was not the case in Experiment 1.

A repeated measures ANOVA was conducted on relative distance error (4 levels of distance $\times 3$ levels of target location $\times 3$ levels of target size $\times 3$ levels of observer position $\times 3$ levels of seating position, with distance, target location, target size, and observer position as withinsubjects factors and seating position as a between-subjects factor). The subjects significantly overestimated distances, with increasing actual distances in the planar area condition, whereas distance estimation errors on uphill and downhill slopes failed to reach significance [planar, $F(3,69)=4.028, p=.047, \varepsilon=.405$; uphill, $F(2,46)=$ $2.728, p=.106, \varepsilon=.574$; downhill, $F(2,46)=1.181$, $p=.316, \varepsilon=.529]$. No effect of observer elevation could be found; contrary to what had been hypothesized, prone positions of the virtual observer (camera) did not lead to farther distance judgments than did upright positions. The size of the targets had a strong effect on distance estimation to the effect that large objects were judged to be closer in planar $[F(2,46)=15.874, p<.001, \varepsilon=.546]$, uphill $[F(2,46)=16.280, p<.001, \varepsilon=.532]$, and downhill $[F(2,46)=14.296, p=.001, \varepsilon=.560]$ terrain. An effect of terrain could not be found; neither did main effects of target location or the observer's seating position in front of the screen emerge. The lack of an effect of seating position is consistent with tolerance toward oblique viewing positions in the cinema (see Cutting, 1987), and it justifies the procedure of having many observers in front of a large projection screen.

Interactions of terrain and distance were found; however, they do not bear on our hypotheses but, rather, on the peculiarities of the natural terrain: terrain $\times$ distance $[F(4,92)=4.608, p=.008, \varepsilon=.656]$ and terrain $\times$ distance $\times$ target location $\times$ target size $[F(16,368)=2.862$, 
Planar Terrain

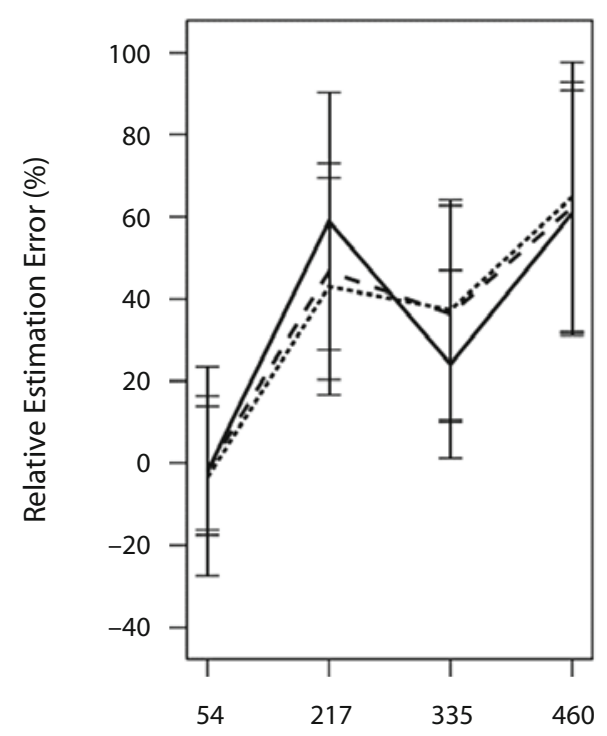

Uphill Terrain

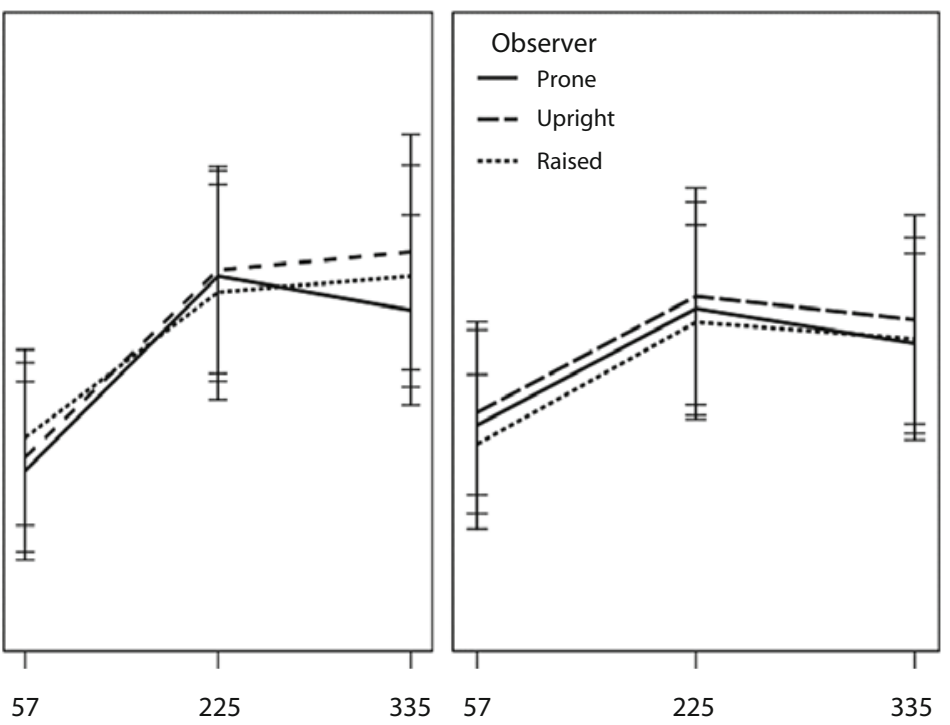

Actual Distance $(\mathrm{m})$

Figure 2. Experiment 2: Average estimation errors expressed in percentages of actual distances for prone, upright, and raised observer positions by planar, uphill, and downhill area. Positive errors indicate overestimation of distance. Error bars represent standard errors of the means.

$p=.037, \varepsilon=.209]$. This could reflect the dip in relative distance estimation error around the middle distances of 225 and $335 \mathrm{~m}$, which was found in the field and in the laboratory. We attribute the dip to terrain peculiarities.

Taken together, the comparatively heterogeneous performance in the laboratory task indicates that important information was lost in the photographic renditions. Presumably, this reduction of useful distance cues induced the observers to focus on retinal size and prompted them to apply a bigger-nearer heuristic. For instance, the target $217 \mathrm{~m}$ in front of a prone virtual observer was judged to be, on average, at $465 \mathrm{~m}$ when small $(0.5 \times 0.5 \mathrm{~m})$, at $302 \mathrm{~m}$ when medium-sized $(0.75 \times 0.75 \mathrm{~m})$, and at a distance of $268 \mathrm{~m}$ when large $(1.0 \times 1.0 \mathrm{~m})$. No such size effect could be observed in the field. In summary, the distances in the laboratory experiment were overestimated to a smaller extent than those in the field experiment. Also, because of the large standard deviations in the laboratory experiment (three to five times higher than those in the field experiment), overestimation was significant only in the planar terrain.

\section{EXPERIMENT 3 \\ Replication of Distance Estimation in Near and Far Vista Space}

Since the laboratory setting of Experiment 2 had produced effects that were not entirely consistent with the field study and that indicated that the lack of resolution may have prompted the observers to use a size-based heuristic, we conducted a second field experiment with the goal of replicating the overestimation found in the first field experiment and testing for effects of observer position in near and far vista space. For this reason, two more distances and a raised observer position were included. We expected estimation error in vista space to grow overproportionally with distance; that is, the farther the object was from the observer, the greater the distance estimation error should become. If present, an effect of observer elevation should be detectable when prone and upright observer postures were supplemented by an observer position that was to the limit of the expected utility of eye height. Finally, by varying target size, we intended to take another look at a potential size-heuristic in the field.

\section{Method}

Observers. Twelve volunteers participated in this experiment. Their ages ranged from 23 to 48 years, with an average of 31 years. Two of the observers were female. All had normal or corrected-tonormal vision and were naive with respect to the purpose of the study.

Stimuli and Design. The experiment was conducted outdoors in a large open field in planar terrain. The field was the same as that used in Experiment 1. No salient landmarks or orientation points were given. Six distances $(27,54,73,97,159$, and $217 \mathrm{~m})$ and three target sizes (small, $0.10 \times 0.10 \mathrm{~m}$; medium-sized, $0.15 \times 0.15 \mathrm{~m}$; large, $0.20 \times 0.20 \mathrm{~m})$ were used, as well as three observer positions (prone, upright, and raised upright by standing on a platform $110 \mathrm{~cm}$ above the ground). Note that for practical reasons, the sizes were smaller than those in Experiment 1. The targets were judged in fully crossed and randomized combinations of size, distance, and observer elevation, amounting to a total of 54 trials per observer.

The targets were square wooden boards, $8 \mathrm{~mm}$ thick, and each fixed on a separate wooden frame of appropriate size. The frame was mounted to a stake that could be driven into the ground. All the targets featured a black painted cross on a white background. For the distance measurements, a Leica LAF 800 Rangemaster laser 
Table 2

Mean Distance Judgments and Standard Deviations

Obtained in the Field (Experiment 3)

\begin{tabular}{|c|c|c|c|c|c|c|c|}
\hline \multirow{3}{*}{$\begin{array}{c}\text { Distance } \\
(\mathrm{m})\end{array}$} & \multirow[b]{3}{*}{ Position } & \multicolumn{6}{|c|}{ Target Size } \\
\hline & & \multicolumn{2}{|c|}{$0.1 \times 0.1$} & \multicolumn{2}{|c|}{$0.15 \times 0.15$} & \multicolumn{2}{|c|}{$0.2 \times 0.2$} \\
\hline & & $M$ & $S D$ & $M$ & $S D$ & $M$ & $S D$ \\
\hline \multirow[t]{3}{*}{27} & Prone & 25.000 & 6.208 & 22.333 & 5.176 & 24.667 & 12.070 \\
\hline & Upright & 22.167 & 4.951 & 21.833 & 5.060 & 20.500 & 5.452 \\
\hline & Raised & 25.583 & 5.632 & 22.667 & 4.030 & 22.583 & 5.712 \\
\hline \multirow[t]{3}{*}{54} & Prone & 68.667 & 41.148 & 70.833 & 29.683 & 44.583 & 15.453 \\
\hline & Upright & 54.333 & 25.314 & 44.833 & 15.590 & 49.167 & 22.771 \\
\hline & Raised & 56.750 & 20.001 & 48.333 & 13.419 & 45.750 & 17.945 \\
\hline \multirow[t]{3}{*}{73} & Prone & 93.417 & 43.933 & 85.083 & 39.486 & 84.917 & 40.410 \\
\hline & Upright & 84.333 & 32.222 & 72.250 & 23.699 & 69.333 & 21.253 \\
\hline & Raised & 76.833 & 28.226 & 79.917 & 49.601 & 82.250 & 37.753 \\
\hline \multirow[t]{3}{*}{97} & Prone & 146.583 & 84.403 & 155.500 & 63.777 & 140.500 & 64.692 \\
\hline & Upright & 103.500 & 28.102 & 112.917 & 49.098 & 122.000 & 35.917 \\
\hline & Raised & 87.500 & 20.817 & 112.333 & 49.731 & 110.417 & 47.647 \\
\hline \multirow[t]{3}{*}{159} & Prone & 294.833 & 97.875 & 298.833 & 166.542 & 235.333 & 80.632 \\
\hline & Upright & 236.667 & 115.253 & 222.833 & 102.749 & 195.917 & 68.811 \\
\hline & Raised & 218.333 & 57.985 & 216.000 & 82.215 & 219.000 & 92.342 \\
\hline \multirow[t]{3}{*}{217} & Prone & 351.333 & 119.181 & 346.000 & 145.052 & 323.333 & 110.563 \\
\hline & Upright & 321.000 & 114.436 & 362.083 & 157.559 & 257.000 & 93.629 \\
\hline & Raised & 328.917 & 144.952 & 303.917 & 106.483 & 303.750 & 84.776 \\
\hline
\end{tabular}

distance finder was used, as well as a compass to keep record of the position of the sun. A Canon Digital Ixus 500 camera was used to document the trials.

Procedure. Two reference objects were placed at a distance of $10 \mathrm{~m}$ in front of the observer. For this reference distance, two blue rain barrels were used, $2 \mathrm{~m}$ apart and $10 \mathrm{~m}$ away from the observer. As before, only in this calibration task did the subjects receive feedback on their estimates of the egocentric distance to the barrels, as well as on the distance between the barrels. It was pointed out that these distances should be used as a standard upon which to base the other judgments. The experimental targets were presented in separate random orders per group. To prevent the observes from exchanging estimates, they were not allowed to talk during the experiment. They wrote each estimate on a separate page. Feedback was not given on any of the experimental trials. After each distance estimate, the observers had to provide a difficulty rating of the estimation on a 10-point rating scale.

The observers were run in four groups of 3 . The targets were presented individually in separate random orders for each group. An assistant positioned and removed each target while the observers had to turn around so they were unable to see the assistant or the target. For trials in the prone observing position, the experimenter put up a visual barrier, rather than turning the observers around, while the targets were exchanged by the assistant.

\section{Results and Discussion}

A repeated measures ANOVA on relative judgment errors ( 6 levels of distance $\times 3$ levels of target size $\times$ 3 levels of observer position) revealed a significant effect of actual distance $[F(5,55)=21.203, p=.001, \varepsilon=$ $.410]$. As is detailed in Table 2, near distances below $70 \mathrm{~m}$ tended to be underestimated, whereas large distance above $70 \mathrm{~m}$ were clearly overestimated. This replicated the results of Experiment 1, albeit with a nearer crossover point from underestimation to overestimation. The effect of eye height was significant; the observers in the prone position judged targets to be farther away than did those in the upright or elevated position $[F(2,22)=12.880, p=$ $.003, \varepsilon=.560]$. This effect was carried by the prone posi- tion, since upright and elevated did not differ significantly (see Figure 3). As before, target size also had a significant main effect $[F(2,22)=5.372, p=.013, \varepsilon=.995]$; smaller targets were consistently estimated to be farther away than larger targets.

The results confirm our hypotheses and emphasize that overestimation of large egocentric distance is to be taken seriously. Certainly, spatial compression is not the rule in large, open, natural environments. Also, the data lend more likelihood to the influence of a bigger-nearer heuristic, and they confirm the importance of a reduction in eye height, which increases overestimation of distance in a manner consistent with the use of perspective cues. The results are consistent with those of Experiment 1, in that the relative distance estimation error increased with distance. However, the overall magnitude of estimated distances was smaller and the crossover point was nearer to the observer than in Experiment 1. A few, less than straightforward explanations for these differences can be entertained. First, all the targets were smaller than before. If a bigger-nearer heuristic was at work, the smaller targets should have prompted the observers to overestimate more. Such overestimation would, in turn, have pushed the crossover point closer, but the data show that overestimations, on the whole, were larger in Experiment 1, in which the targets were five times larger. Second, it is conceivable that the calibration procedures used in the two experiments were to blame. In Experiment 1, we arranged stacks of tires to be $10 \mathrm{~m}$ away from the observer and $2 \mathrm{~m}$ apart from one another; in Experiment 2, for lack of tires, we positioned rain barrels in the same manner. It is possible that the observers took the tires as belonging to tractors rather than to cars, which they did. Third, the boundaries of the terrain were different. Whereas in Experiment 1 the targets were placed up to the border of the experimental area, next to surrounding trees in the back- 


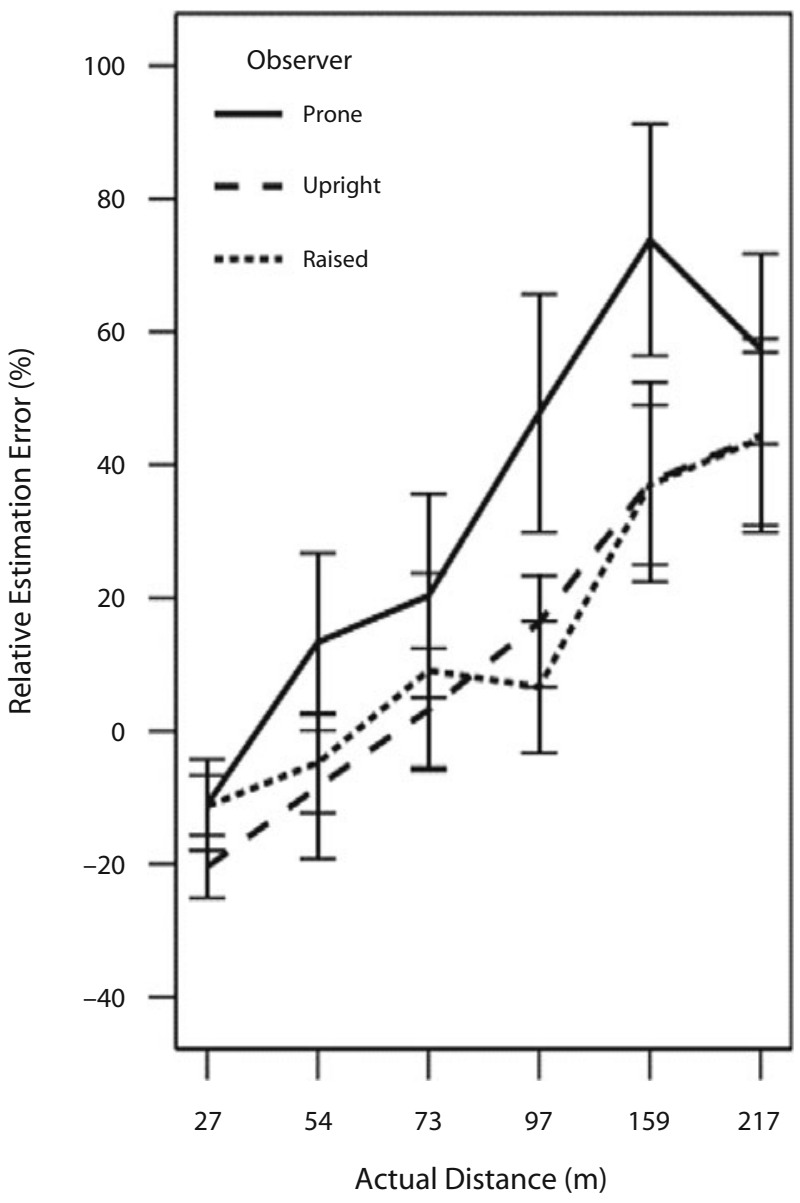

Figure 3. Experiment 3: Average estimation expressed in percentages of actual distance for prone, upright, and raised observer positions. Error bars represent standard errors of the means.

ground, in the present experiment, the targets remained in the first third of that area, keeping the trees well beyond. The unusually large overestimations of the far targets in Experiment 1 may have been related to their proximity to the background. Another potential explanation could be sought in the additional raised observer condition, which was present in Experiment 2 only and which may have scaled down the overall distances. In sum, it seems that the cues provided by the background trees in Experiment 1 can provide the most likely explanation for the differences.

\section{GENERAL DISCUSSION}

Taken together, the experiments revealed that observers do make systematic distance estimation errors in large open environments. Contrary to common belief, distances are not universally underestimated once the realm of informative stereopsis and vergence information is exceeded. Instead, depending on the egocentric region of space, distance underestimation or overestimation can be found. When Grüsser's (1983) model of extrapersonal space is applied, estimates in personal and action space are rather veridical, whereas distances in near vista space are compressed - in particular, when indirect metrics are used, such as the task to equate frontoparallel and sagittal distances. In far vista space, however, there comes a point where compression gives way to an expansion or dilation of subjective space. We found that in far vista space, starting somewhere beyond 75 or $100 \mathrm{~m}$ from the observer, distances are overestimated. This finding is in keeping with some of the first ecological studies on the perception of very large distances (e.g., Purdy \& Gibson, 1955), and it is also consistent with the conventional wisdom of experts, such as marksmen.

\section{The Informational Basis for \\ Distance Estimation in Vista Space}

Any systematic distance estimation errors in vista space have to be evaluated with a number of caveats. For one, estimates become more variable the farther the target is from the observer. Second, the systematic compression and dilation effects reported here exist on top of a number of pictorial effects. For instance, the change in perspective gradient or texture compression caused by lowering the observer's eye height led to overestimation of distance. Furthermore, the retinal size of the target mattered consistently; smaller targets were estimated to be farther away than larger targets. Both findings suggest that some simple heuristics may be at work when large distances are judged, such as texture compression indicates large distance or retinally larger objects must be nearer than retinally smaller objects.

Another caveat concerns the estimation method that is applied. We have used an egocentric distance estimation task. The spatial representation underlying a blindwalking task or an equal distance task is known to produce different results, and matching a frontoparallel distance with a given sagittal distance leads to a foreshortening in the sagittal direction (e.g., Amorim et al., 1998; Loomis et al., 1996). In light of these method effects, a replication of the present experiments with allocentric distance judgments seems desirable.

Also, the role of experience on distance estimation in far vista space remains to be investigated. E. J. Gibson, Bergman, and Purdy (1955) showed that preliminary training with a distance scale improved absolute estimation of distance to an unfamiliar target in a different locale. The training also reduced the standard deviations from pre- to posttest, with a tendency to decrease even with very far distances. We used their findings to motivate our procedure of presenting observers with a calibration anchor in all the experiments.

Given that, in vista space, the entire list of classical monocular cues to distance is likely to contribute to a given distance estimate, it will not be easy to determine the exact informational basis for estimates in far vista space. We have shown that some exemplar cues, such as texture compression, perspective, and retinal size, were incorporated into the distance estimate regardless of whether the target was in near or in far vista space. It is thus likely that the crossover between compression and dilation at about 
$100 \mathrm{~m}$ is brought about not by the disuse of one or another distance cue but, rather, by a reweighting of the cues. At this point, we do not know which particular cue receives a larger or smaller weight.

\section{Reconceiving Vista Space}

Notwithstanding the effects of perspective and retinal size, which seem to permeate all distance estimation in vista space, quite surprisingly, a crossover point exists from overall compression to dilation of perceptual space around $100 \mathrm{~m}$. Distances of targets nearer than $100 \mathrm{~m}$ are underestimated, whereas distances above $100 \mathrm{~m}$ are overestimated, and increasingly so as they recede into far vista space. This result not only confirms Gibson's initial findings (E. J. Gibson \& Bergman, 1954; Purdy \& Gibson, $1955)$, but also suggests that vista space is not uniform. In our opinion, Grüsser's (1983) and Cutting and Vishton's (1995) subdivision of space has to be specified by one further division: Vista space has to be differentiated into near and far vista space. Near vista space up to $100 \mathrm{~m}$ behaves differently than far vista space beyond $100 \mathrm{~m}$. The exact position of the crossover point may vary; it seems to depend on the size of the targets, as well as on observer and environmental conditions. Underestimation and spatial compression in near vista space may be roughly proportional, whereas overestimation and expansion of the visual field in far vista space appear to be overproportional. We realize that the claim of overproportionality of overestimation rests on weak grounds, because we were confined to using relatively few distance settings between 25 and $460 \mathrm{~m}$ in the large open field. The exact curve of the distance overestimation remains to be determined. The same holds for the role of hollows and uphill and downhill slopes. We also do not know if the overestimation is best thought of as an expansion of perceptual space or as a misperception of the slope of the ground plane. However, our results have ruled out the possibility that the slope of ground plane is uniformly overestimated, as may be the case for near vista space (Ooi, Wu, \& He, 2006).

The subdivision of vista space does lend support to anecdotal reports by experts who advise on how to reckon with overestimation of very large distances. The practical implications in the military realm are obvious, especially in the context of target shooting (Watson, 1982, pp. 69ff). Joast (1984, p. 644) described the determination of the correct distance as one of the greatest problems in the use of firearms. Even at "easy" distances below $300 \mathrm{~m}$, targets are missed as a consequence of distance overestimation. With a correct distance estimate, marksmen can deliver precise shots over distances up to $1,000 \mathrm{~m}$, and mortar or artillery fire is often aimed at even farther targets (Rogers, 1994, pp. 29ff). Needless to say, the perceptual biases are likely to become exacerbated when viewing conditions deteriorate; for instance, when the visual field is constrained, vision is available only when mediated by a mirror or when a night vision device is in use.

In essence, then, we conclude that estimates of large distances have to be treated as a phenomenon in their own right. They cannot be handled within the same theoretical framework as that appropriate for distances in action space or in near vista space. Reports of distance overestimation in vista space have to be taken seriously. As a consequence of our results, the notion of vista space has to be reconceived. It can no longer be thought of as a uniform space that is somewhat compressed. Instead, it has to be divided into two distinct regions. Near vista space, up to $100 \mathrm{~m}$ from the observer, remains associated with the traditional aspects of vista space. Far vista space, on the other hand, has different properties. It remains to be explored what these properties are above and beyond the tendency to dilate space that we have documented here.

\section{AUTHOR NOTE}

We thank the fire department of Rennerod and Helmut Scherer from the forest department of Lasterbach for their technical support. Jürgen Bußweiler helped manufacture the targets. Bianca Abel, Jan-Eric Daum, and Sebastian Daum assisted during data collection in the field experiments. Matthias Gamer gave statistical, and Agnes Münch computer advice. Joel Norman provided insightful comments on an earlier version of the manuscript. Petra Glaubitz assisted in administering the questionnaires. Evgeniya Boyarskaya gave helpful support during the revision of the article. We also thank all the volunteers who made this study possible. Correspondence concerning this article should be addressed to H. Hecht, Psychologisches Institut, Johannes Gutenberg-Universität Mainz, 55099 Mainz, Germany (e-mail: hecht@uni-mainz.de).

Note-Accepted by the previous editorial team, when Thomas H. Carr was Editor.

\section{REFERENCES}

Allekseenko, V. (1989). Nepreryvno, tschtschantel'no i svoevremenno [Principles of conducting reconnaissance]. Voennyj Vestnik, 5, 42-44. Amorim, M. A., Loomis, J. M., \& FukUsima, S. S. (1998). Reproduction of object shape is more accurate without the continued availability of visual information. Perception, 27, 69-86.

ANDRe, J., \& Rogers, S. (2006). Using verbal and blind-walking distance estimates to investigate the two visual systems hypothesis. Perception \& Psychophysics, 68, 353-361.

BADEN-Powell, R. S. S. (1944). Scouting for boys. London: Pearson.

BeE, L. (1991). Effect of magnification on distance estimation. Farnborough, U.K.: Army Personnel Research Establishment, Ministry of Defence.

Bhalla, M., \& Proffitt, D. R. (1999). Visual-motor recalibration in geographical slant perception. Journal of Experimental Psychology: Human Perception \& Performance, 25, 1076-1096.

Bressan, P., Garlaschelli, L., \& Barracano, M. (2003). Antigravity hills are visual illusions. Psychological Science, 14, 441-449.

Creem, S. H., \& Proffitt, D. R. (1998). Two memories for geographical slant: Separation and interdependence of action and awareness. Psychonomic Bulletin \& Review, 5, 22-36.

CutTing, J. E. (1987). Rigidity in cinema seen from the front row, side aisle. Journal of Experimental Psychology: Human Perception \& Performance, 13, 323-334.

Cutting, J. E., \& Vishton, P. M. (1995). Perceiving layout and knowing distances: The integration, relative potency, and contextual use of different information about depth. In W. Epstein \& S. Rogers (Eds.), Handbook of perception and cognition: Vol. 5. Perception of space and motion (pp. 69-117). San Diego: Academic Press.

DA SILVA, J. A. (1985). Scales for perceiving egocentric distance in a large open field: Comparison of three psychophysical methods. American Journal of Psychology, 98, 119-144.

FINE, B. J., \& Kobrick, J. L. (1983). Individual differences in distance estimation: Comparison of judgments in the field with those from projected slides of the same scenes. Perceptual \& Motor Skills, 57, 3-14.

Galanter, E., \& Galanter, P. (1973). Range estimates of distant visual stimuli. Perception \& Psychophysics, 14, 301-306.

Gibson, E. J., \& Bergman, R. (1954). The effect of training on absolute estimation of distance over the ground. Journal of Experimental Psychology, 48, 473-482. 
Gibson, E. J., Bergman, R., \& Purdy, J. (1955). The effect of prior training with a scale of distance on absolute and relative judgments of distance over the ground. Journal of Experimental Psychology, 50, $97-105$.

GiBson, J. J. (1950). The perception of visual surfaces. American Journal of Psychology, 63, 367-384.

Gibson, J. J. (1979). The ecological approach to visual perception. Hillsdale, NJ: Erlbaum.

Gibson, J. J., \& Connsweet, J. (1952). The perceived slant of visual surfaces-Optical and geographical. Journal of Experimental Psychology, 44, 11-15.

GibSON, J. J., \& FLOCK, H. (1962). The apparent distance of mountains. American Journal of Psychology, 75, 501-503.

GiLINSKY, A. S. (1951). Perceived size and distance in visual space. Psychological Review, 58, 460-482.

GoGEL, W. C. (1964). Visual perception of spatial extent. Journal of the Optical Society of America, 54, 411-416.

GrüsSER, O. J. (1978). Grundlagen der neuronalen Informationsverarbeitung in den Sinnesorganen und im Gehirn. In S. Schindler \& W. K. Giloi (Eds.), Informatik fachberichte (Vol. 16, pp. 234-273). Berlin: Springer.

GRÜSSER, O. J. (1983). Multimodal structure of the extrapersonal space. In A. Hein \& M. Jeannerod (Eds.), Spatially oriented behavior (pp. 327-352). New York: Springer.

HauCK, G. (1875). Die subjektive Perspektive und die horizontalen Curvaturen des Dorischen Stils [Subjective perspective and the horizontal curvatures of the Doric style]. Stuttgart: Wittwer.

Hecht, H., van Doorn, A., \& Koenderink, J. J. (1999). Compression of visual space in natural scenes and in their photographic counterparts. Perception \& Psychophysics, 61, 1269-1286.

Higashiyama, A. (1996). Horizontal and vertical distance perception: The discorded-orientation theory. Perception \& Psychophysics, 58, 259-270.

Higashiyama, A., \& Ueyama, E. (1988). The perception of vertical and horizontal distances in outdoor settings. Perception \& Psychophysics, 44, 151-156.

Hutchinson, J. J., \& LoOMIs, J. M. (2006a). Does energy expenditure affect the perception of egocentric distance? A failure to replicate Experiment 1 of Proffitt, Stefanucci, Banton, and Epstein (2003). Spanish Journal of Psychology, 9, 332-339.

Hutchinson, J. J., \& Loomis, J. M. (2006b). Reply to Proffitt, Stefanucci, Banton, and Epstein. Spanish Journal of Psychology, 9, 343-345.

Ivanov, A. (1969). O metodike obucebija glazomeru [The methods for distance estimation training]. Vojennyj Vestnik, 3, 114-116.

JiANG, Y., \& MARK, L. S. (1994). The effect of gap depth on the perception of whether a gap is crossable. Perception \& Psychophysics, 56, 691-700.

JoAST, S. (1984). Scharfschützen (II): Auswahl, Ausbildung, Einsatz. Truppendienst, 23, 644-648.

KammanN, R. (1967). The overestimation of vertical distance and slope and its role in the moon illusion. Perception \& Psychophysics, 2, 585-589.

Kuroda, T. (1971). Distance constancy: Functional relationships between apparent distance and physical distance. Psychologische Forschung, 34, 199-219.

Lappin, J. S., Shelton, A. L., \& Rieser, J. J. (2006). Environmental context influences visually perceived distance. Perception \& Psychophysics, 68, 571-581.

Loomis, J. M., Da Silva, J. A., Fujita, N., \& Fukusima, S. S. (1992). Visual space perception and visually directed action. Journal of Experimental Psychology: Human Perception \& Performance, 18, 906-921.

Loomis, J. M., Da Silva, J. A., Philbeck, J. W., \& Fukusima, S. S. (1996). Visual perception of location and distance. Current Directions in Psychological Science, 5, 72-77.
LuKas, J. (1996). Psychophysik der Raumwahrnehmung [The psychophysics of space perception]. Weinheim: Beltz Psychologie.

Norman, J. F., Todd, J. T., Perotti, V. J., \& Tittle, J. S. (1996). The visual perception of three-dimensional length. Journal of Experimental Psychology: Human Perception \& Performance, 22, 173-186.

OKaвe, A., Аокі, K., \& Намамото, W. (1986). Distance and direction judgment in a large scale natural environment: Effects of a slope and winding trial. Environment \& Behavior, 18, 755-772.

OoI, T. L., Wu, B., \& HE, Z. J. (2006). Perceptual space in the dark affected by the intrinsic bias of the visual system. Perception, 35, 605-624.

Plumert, J. M., Kearney, J. K., Cremer, J. F., \& Recker, K. (2005). Distance perception in real and virtual environments. ACM Transactions on Applied Perception, 2, 216-233.

Proffitt, D. R., Bhalla, M., Gossweiler, R., \& Midgett, J. (1995). Perceiving geographical slant. Psychonomic Bulletin \& Review, 2 , 409-428.

Proffitt, D. R., Stefanucci, J., Banton, T., \& Epstein, W. (2003). The role of effort in perceiving distance. Psychological Science, 14, 106-113.

Proffitt, D. R., Stefanucci, J., Banton, T., \& Epstein, W. (2006a). A final reply to Hutchinson and Loomis. Spanish Journal of Psychology, 9, 346-348.

Proffitt, D. R., Stefanucci, J., Banton, T., \& Epstein, W. (2006b). Reply to Hutchinson and Loomis. Spanish Journal of Psychology, $\mathbf{9}$, 340-342.

PuRdY, J., \& GibSon, E. J. (1955). Distance judgement by the method of fractionation. Journal of Experimental Psychology, 50, 374-380.

Rogers, A. (1994). Scharfschützenausbildung bei den Royal Marines. Barett, 5, 29-34.

Ross, H. E. (1967). Water, fog and the size-distance invariance hypothesis. British Journal of Psychology, 58, 301-311.

Ross, H. E. (1974). Behaviour and perception in strange environments. London: George Allen \& Unwin.

Ross, H. E. (1975). Mist, murk and visual perception. New Scientist, 19, 658-660.

Ross, H. E., \& Plug, C. (2002). The mystery of the moon illusion: Exploring size perception. Oxford: Oxford University Press.

SinAI, M. J., OoI, T. L., \& He, Z. J. (1998). Terrain influences the accurate judgment of distance. Nature, 395, 497-500.

StockFIsch, D. (2006). Der Reibert: Das Handbuch für den deutschen Soldaten. Hamburg: Mittler \& Sohn.

Teghtsoonian, R., \& Teghtsoonian, M. (1970). Scaling apparent distance in a natural outdoor setting. Psychonomic Science, 21, 215-216.

Thompson, W. B., Willemsen, P., Gooch, A. A., \& Creem-Regehr, S. H. (2004). Does the quality of the computer graphics matter when judging distances in visually immersive environments? Presence, $\mathbf{1 3}$, $560-571$.

ToYe, R. C. (1986). The effect of viewing position on the perceived layout of space. Perception \& Psychophysics, 40, 85-92.

Wagner, M. (1985). The metric of visual space. Perception \& Psychophysics, 38, 483-495.

Watson, P. (1982). Psychokrieg: Möglichkeiten, Macht und Mißbrauch der Militärpsychologie [War on the mind]. Frankfurt am Main: Fischer Taschenbuch.

Witt, J. K., Proffitt, D. R., \& Epstein, W. (2004). Perceiving distance: A role of effort and intent. Perception, 33, 577-590.

Wraga, M., \& Proffitt, D. R. (2000). Mapping the zone of eye-height utility for seated and standing observers. Perception, 29, 1361-1383.

Wu, B., OoI, T. L., \& He, Z. J. (2004). Perceiving distance accurately by a directional process of integration ground information. Nature, 428, 73-77.

(Manuscript received May 25, 2007; revision accepted for publication February 20, 2009.) 